\title{
Systems Biology: The take, input, vision, concerns and hopes
}

\author{
Graeme Tucker ${ }^{1}$ \\ ${ }^{1}$ Lighthouse Laboratories, SABC Loneragan Building, Murdoch University, South Street, Murdoch, Western Australia 6150. Correspondence should be ad- \\ dressed to Graeme Tucker (gtucker@lighthouselabs.org.au). \\ Received Jul. $4^{\text {th }}, 2008$; revised Mar. $1^{\text {st }}, 2009$; accepted Mar. $9^{\text {th }}, 2009$
}

\begin{abstract}
Systems Biology is a relatively new branch of biology that brings together an interdisciplinary team of scientist, computer engineers and mathematicians. Biomedicine can gain much from the input of Systems Biology. The object and aims of this article centre on clarification and direction for Systems Biology, notably in regard to human health and disease.
\end{abstract}

The take: Many consider today's Systems Biology as an incarnation that was instigated by the biotechnological revolution of high throughput data acquiring instruments. Data gathering and processing, emerged with two streams: refinement during the gathering stage or, refinement at the point of analysis. However, the rapid development of this high throughput hardware was missing the resources to decipher the vast amounts of data produced. Handling of such data required the adaptation of computational platforms and users. The user(s) emerged as an interdisciplinary cross breed of researchers: mathematicians, computer specialist and to a lesser extent the biologist, because of skill displacement. An immediate challenge for this new breed of researcher was defining Systems Biology. Initial workers reflected upon their activity for inspiration: informatics /computational biology demonstrates the 'relativity' of biological composite by use of 'networking' illustrations supported by software programs and mathematics. Consideration with regard to this form of data representation closely resembled their engineering heritage; in particular Control Systems. Thus, the word 'System' had relevance. Because the theme related to Biology the phrase Systems Biology was coined.

However, a decade on there is still controversy surrounding the definition of Systems Biology and what it means to a broad spectrum of researchers in their daily activities. Rightly or wrongly the biologist and philosopher is at risk of being displaced from the driving seat of biological research. Computology could function as a new word to describe the computer and mathematical specialist engaged in biology. Aside from those resisting change, many traditional biologist have reacted to this branch of biology as a branch lacking turgidity. Others argue that their wet medium for research is now waters muddied by Systems Biology. Advocates of Systems Biology from all camps continue to seek clarification and as such new definitions are regularly floated as vessels to carry Systems Biologists on their voyage of discovery.

Input: In a diffuse setting Systems Biology in one sentence could be described as follows: 'Systems Biology is a branch of biology that is an interjection of fields and disciplines, with applications collectively integrating broadly acquired data from all research levels for the purpose of indicating or modelling phenotype.'

A definition of Systems Biology in one word could possibly be, 'Relativity'. Nature complies with the law of physics and physical things are superimposed on mathematical principles. This can include stimuli such as the fight or flight response or, sexual arousal.

Of particular interest and relevance, is the notion that the Systems Biologist will consider and address their challenge beyond the immediate viewpoint of the biological composites of interest.

The term 'Model' is frequently used in context of Systems Biology. A model is a representation of a feature/function; a condition that is simulated or, a composite that is mimicked. That representation need not necessarily be a biological attribute. For example an intrinsic or extrinsic stressor (infection or sun stroke) can lead directly to a physical affect (fever or hyperthermia).. In an indirect setting such as arachnophobia, consideration is given to memory, psychological predisposition and reflex. An example of a traditional model for a biologist is an organism such as yeast or, the mouse. A model for the computer engineer is a software program; a model for a mathematician is an equation. Thus, computation serves to 'simulate,' mathematics serves to 'mimic' and biology serves to 'feature.' Together, they serve Systems Biology. From a retrospective perspective the phrase is perceived as an encompassing mode or stage. From another perspective, Systems Biology is a language, a script and an instrument. In many respects it is the mirrored reflection of Biological Systems in the literal sense.

Vision: In the not too distant future it is suspected that 
Systems Biology will be a prerequisite of project planning for wet based laboratory research, much in the same way that consideration of ethics is a necessity for research endorsement and support.

Concerns: Wet laboratory research has had the in vitro model for many years. For the researcher it has proved unambiguous in distinguishing the significance and relativity of outcomes to that of the in vivo setting. However, with the emergence of the in silico model the risk of ambiguity is clearly present. Of particular concern is that wet lab 'fact' is fiction-ated (in silico) and that the subsequent fiction outcomes, after model manipulation / extrapolation, are inferred as fact in the bigger picture of true fact. In that setting the audience of communicated in silico science is at risk of having their own works misguided, notably the wet laboratory researcher. Therefore is the informatics camp muddying the waters of wet lab science?

In addition, is in silico science operating with aseptic techniques when fact-ors are introduced in the extrapolation process? Is there a risk of fashioning fact to the point of becoming arte-fact?

Equally, is there a risk of fashioning wet laboratory experiments to fit the predicted in silico assumptions?

Hopes: Systems Biology needs some standardisation policies and techniques to ensure that the concerns outlined above are not encountered.

The incorporation of Systems Biology principles in project planning will hopefully have the benefit of minimising and reducing calculated aspects such as cost and risk. However, in the spirit of far reaching science (true philosophy), which is usually accompanied by risk, it is hoped that this reach is not compromised; if anything, it is hoped that Systems Biology will gather the pace and direction of research.

Turnaround benefits shall hopefully be seen in medicine (facilitation of modes for the prevention, diagnosis, treatment and cure of disease), agriculture (increased crop yield, crop resistance and crop tolerance) and in the development of sustainable energy through bio-fuels.

Beyond Systems Biology is Systems Life. Can it factor in consciousness and meaning? 